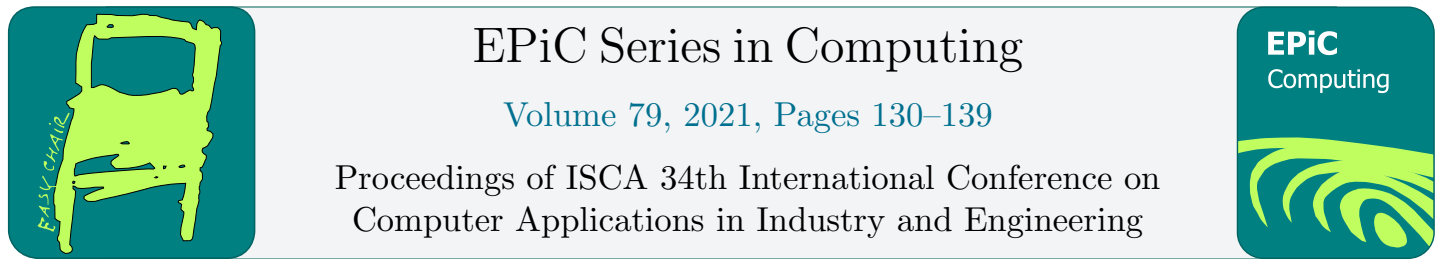

\title{
Analysis and Control of Linear Time-Varying (LTV) Systems
}

Robert N. K. Loh ${ }^{1}$ and K. C. Cheok ${ }^{2}$

${ }^{1,2}$ Oakland University, Rochester, MI, 48309-4401, USA

loh@oakland.edu, cheok@oakland.edu

\begin{abstract}
Consider a linear time-varying (LTV) system described by the state-space equation $\dot{\mathbf{x}}=\mathbf{A}(t) \mathbf{x}+\mathbf{B}(t) \mathbf{u}(t)$. The main objectives of this paper include: (i) determination of the analytical (closed-form) solutions for the fundamental matrix $\mathbf{X}(t)$ and the state transition matrix $\boldsymbol{\Phi}\left(t, t_{o}\right)$ of the LTV system, and (ii) design of feedback control, such that the closed-loop system matrix $\mathbf{A}_{c l}(t)=\mathbf{A}(t)-\mathbf{B}(t) \mathbf{K}(t)$, where $\mathbf{K}(t)$ is a gain matrix, has desirable -properties, namely, $\mathbf{A}_{c l}(t)$ is commutative and triangular, thereby analytical solutions for $\mathbf{X}_{c l}(t)$ and $\mathbf{\Phi}_{c l}\left(t, t_{o}\right)$ can be determined. This is different from a standard pole-placement design problem where the goal is to place the poles of $\mathbf{A}_{c l}(t)$ at desired locations for achieving desired closed-loop stability properties. Examples are given to demonstrate the design objectives. Solutions in Matlab are given as well.
\end{abstract}

\section{Linear-Time-Varying (LTV) Systems}

Consider an $n$ th-order linear time-varying (LTV) system described by the state-space equation:

$$
\dot{\mathbf{x}}=\mathbf{A}(t) \mathbf{x}+\mathbf{B}(t) \mathbf{u}(t), \quad \mathbf{x}\left(t_{o}\right)=\mathbf{x}_{o}, \quad t_{o} \in[a, b],
$$

where $\mathbf{x}(t)$ is an $n \times 1$ state vector, and $\boldsymbol{u}(t)$ is an $\ell \times 1$ control vector, $\mathbf{A}(t)$ and $\mathbf{B}(t)$ are, respectively, $n \times n$ and $n \times \ell$ time-varying matrices, and $\mathbf{x}\left(t_{o}\right)=\mathbf{x}_{o}$ is the initial condition. By the method of variation of parameters, the solution of (1) can be expressed as

$$
\mathbf{x}(t)=\boldsymbol{\Phi}_{A}\left(t, t_{o}\right) \mathbf{x}\left(t_{o}\right)+\int_{t_{o}}^{t} \boldsymbol{\Phi}_{A}(t, \tau) \mathbf{B}(\tau) \mathbf{u}(\tau) d \tau,
$$

where $\boldsymbol{\Phi}_{A}\left(t, t_{o}\right)$ denotes the $n \times n$ state transition matrix associated with $\mathbf{A}(t)$, and satisfies

$$
\dot{\boldsymbol{\Phi}}_{A}\left(t, t_{o}\right)=\mathbf{A}(t) \boldsymbol{\Phi}_{A}\left(t, t_{o}\right), \quad \boldsymbol{\Phi}_{A}\left(t_{0}, t_{o}\right)=\mathbf{I}_{n},
$$

where $\mathbf{I}_{n}$ denotes the $n \times n$ unit matrix. The transition matrix $\boldsymbol{\Phi}_{A}(t, \tau)$ is related to the fundamental matrix $\mathbf{X}_{A}(t)$ by, 


$$
\mathbf{\Phi}_{A}(t, \tau)=\mathbf{X}_{A}(t) \mathbf{X}_{A}^{-1}(\tau),
$$

where the fundamental matrix

$$
\mathbf{X}_{A}(t)=\exp \left(\int_{t_{o}}^{t} \mathbf{A}(\tau) d \tau\right)
$$

solves the $n \times n$ matrix differential equation

$$
\dot{\mathbf{X}}_{A}(t)=\mathbf{A}(t) \mathbf{X}_{A}(t), \quad \mathbf{X}_{A}\left(t_{0}\right)=\mathbf{I}_{n} .
$$

Further, the matrix exponential in (5) is defined by the power series $\sum_{k=0}^{\infty} \frac{1}{k !}\left(\int_{t_{o}}^{t} \mathbf{A}(\tau) d \tau\right)^{k}$, thereby (5) yields, in general, a solution of the form (an infinite-sum solution)

$$
\mathbf{X}_{A}(t)=\exp \left(\int_{t_{o}}^{t} \mathbf{A}(\tau) d \tau\right)=\sum_{k=0}^{\infty} \frac{1}{k !}\left(\int_{t_{o}}^{t} \mathbf{A}(\tau) d \tau\right)^{k}
$$

However, if $\left(\int_{t_{o}}^{t} \mathbf{A}(\tau) d \tau\right)^{m+1}=\mathbf{0}$ for some finite $m<\infty$, then (7) becomes a finite-sum solution [1]

$$
\mathbf{X}_{A}(t)=\mathbf{I}_{n}+\int_{t_{o}}^{t} \mathbf{A}(\tau) d \tau+\cdots+\frac{1}{m !}\left(\int_{t_{o}}^{t} \mathbf{A}(\tau) d \tau\right)^{m} .
$$

It is well known that determining the matrix exponential given by (5) is a difficult task, even for constant matrix $\mathbf{A}$ [2]. Note also that $\mathbf{X}_{A}(t)$ is nonsingular for all $t$, but may be nonunique; however, the state transition matrix given by $\mathbf{\Phi}_{A}\left(t, t_{o}\right)=\mathbf{X}_{A}(t) \mathbf{X}_{A}^{-1}\left(t_{o}\right)$ is unique for all $t_{o}$ and $t$.

\section{Facts about LTV Systems}

Consider an LTV system described by the homogenous ordinary differential equation (ODE)

$$
\dot{\mathbf{x}}=\mathbf{A}(t) \mathbf{x}, \quad \mathbf{x}\left(t_{o}\right)=\mathbf{x}_{o} .
$$

The following are important facts about the fundamental matrix $\mathbf{X}_{A}(t)$ and state transition matrix $\boldsymbol{\Phi}_{A}\left(t, t_{o}\right)$ associated with $\mathbf{A}(t)$ :

\section{F1: Sufficient Conditions for the Existence of Fundamental and State Transition Matrices}

The conditions are summarized in Table 1 ([3], [4], [5], [6, Th 2.1], [7], [8, Th 5.5], [9, Th 2,]):

Table 1: Sufficient Conditions for the Existence of Analytical Solutions of $\mathbf{X}_{A}(t)$ and $\boldsymbol{\Phi}_{A}\left(t, t_{o}\right)$ :

(i) $\quad \mathbf{A}(\tau)$ has piecewise continuous elements $\left\{a_{i j}(\tau)\right\}$ for all $i, j$ and $\tau \in\left[t_{o}, t\right]$;

(ii) $\mathbf{A}(t)$ commutes with its integral $\int_{t_{o}}^{t} \mathbf{A}(\tau) d \tau$, i.e., $\mathbf{A}(t)\left(\int_{t_{o}}^{t} \mathbf{A}(\tau) d \tau\right)=\left(\int_{t_{o}}^{t} \mathbf{A}(\tau) d \tau\right) \mathbf{A}(t)$;

(iii) $\mathbf{A}\left(t_{1}\right) \mathbf{A}\left(t_{2}\right)=\mathbf{A}\left(t_{2}\right) \mathbf{A}\left(t_{1}\right)$ for all $t_{1}$ and $t_{2}$;

(iv) $\int_{t_{o}}^{t_{1}} \mathbf{A}(\tau) d \tau \int_{t_{o}}^{t_{2}} \mathbf{A}(s) d s=\int_{t_{o}}^{t_{2}} \mathbf{A}(s) d s \int_{t_{o}}^{t_{1}} \mathbf{A}(\tau) d \tau$;

(v) $\quad \mathbf{A}(t)=\beta(t) \mathbf{A}$, where $\beta(t)$ is a scalar function and $\mathbf{A}$ is a constant matrix; 
(vi) $\quad \mathbf{A}(t) \quad$ can be diagonalized as $\mathbf{D}(t)=\mathbf{T}^{-1} \mathbf{A}(t) \mathbf{T}$, where $\mathbf{D}(t)=\operatorname{diag}\left(\lambda_{1}(t), \ldots, \lambda_{n}(t)\right) \quad$ and $\left\{\lambda_{1}(t), \ldots, \lambda_{n}(t)\right\}$ denote the eigenvalues of $\mathbf{A}(t)$.

Since the above are only sufficient conditions, the fundamental and state transition matrices of $\dot{\mathbf{x}}=\mathbf{A}(t) \mathbf{x}$ may exist even when those conditions are not met. Note that (i) - (vi) hold for linear timeinvariant (LTI) systems, and are applicable when $\mathbf{A}(\mathrm{t})$ is replaced by $\mathbf{A}_{c l}(t) \triangleq[\mathbf{A}(t)-\mathbf{B}(t) \mathbf{K}(t)]$.

\section{F2: Method of Superposition Principle (MSP)}

A $n \times n$ analytical solution $\mathbf{X}(t)$ for the homogenous LTV system described by ODE (6) can be constructed by picking $n$ linearly independent initial conditions [4], [10], [11 ]. The method is summarized as follows:

Set $n$ normalized independent initial condition (IC) as:

$$
\mathbf{x}^{1}\left(t_{o}\right)=\left[\begin{array}{c}
1 \\
0 \\
\vdots \\
0
\end{array}\right], \mathbf{x}^{2}\left(t_{o}\right)=\left[\begin{array}{c}
0 \\
1 \\
\vdots \\
0
\end{array}\right], \cdots, \mathbf{x}^{n}\left(t_{o}\right)=\left[\begin{array}{c}
0 \\
0 \\
\vdots \\
1
\end{array}\right] .
$$

Let $\left\{\mathbf{x}^{i}(t), i=1, \cdots, n\right\}$ be the solution of $\dot{\mathbf{x}}=\mathbf{A}(t) \mathbf{x}$ based on (10), i.e., $\mathbf{x}^{i}(t)$ is solved uniquely one column at a time for each initial condition $\mathbf{x}^{i}\left(t_{o}\right)$. This construction is based on the principle of superposition of linear systems, and is a method of superposition principle (MSP) with normalized ICs. The resulting $n \times n$ nonsingular matrix, denoted by $\mathbf{X}_{\text {Normalized }}(t)$, is given by

$$
\mathbf{X}_{\text {Normalized }}(t)=\left[\begin{array}{l:l|l}
\mathbf{x}^{1}(t) & \cdots & \mathbf{x}^{n}(t)
\end{array}\right] .
$$

It follows from (9) and (11) that the normalized fundamental matrix $\mathbf{X}_{\text {Normalized }}(t)$ satisfies

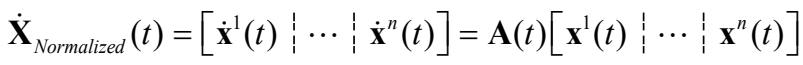

$$
\begin{aligned}
& =\mathbf{A}(t) \mathbf{X}_{\text {Normalized }}(t) \text {. }
\end{aligned}
$$

Note that given $\dot{\mathbf{x}}=\mathbf{A}(t) \mathbf{x}$, it is convenient to choose its initial condition given by (10). However, it is also possible to choose any set of initial conditions $\left\{\overline{\mathbf{x}}^{i}\left(t_{o}\right), i=1,2, \cdots, n\right\}$, thereby obtaining a different $\overline{\mathbf{X}}(t)$, as long as $\left\{\overline{\mathbf{x}}^{i}\left(t_{o}\right), i=1,2, \cdots, n\right\}$ are linearly independent vectors. Therefore, the non-uniqueness of $\mathbf{X}_{\text {Normalized }}(t)$ and $\overline{\mathbf{X}}(t)$ provides flexibilities for analyzing LTV systems; however, the state transition matrix $\mathbf{\Phi}\left(t, t_{o}\right)$ is unique and is given by (4).

Remark 1: The method of superposition principle (MSP) hinges on $\mathbf{A}(t)$ being a triangular matrix, for example, a lower triangular $\mathbf{A}(t)=\left[\begin{array}{cc}a_{11}(t) & 0 \\ a_{21}(t) & a_{22}(t)\end{array}\right]$ or upper triangular $\mathbf{A}(t)=\left[\begin{array}{cc}a_{11}(t) & a_{12}(t) \\ 0 & a_{22}(t)\end{array}\right]$, so that the analytical solution of each ODE $\dot{x}_{i}=\sum_{j=1}^{n} a_{i j}(t) x_{j}$ for all $i=1,2, \ldots, n$ and $j=1,2, \ldots, n$ can be determined without regard to the matrix commutativity of $\mathbf{A}(t)$. The method is applicable to triangular 
systems of any order $n$, and are particularly attractive for manual calculations of $\mathbf{X}(t)$ and $\mathbf{\Phi}\left(t, t_{o}\right)$ for low-dimensional systems, such as for $n=2$ and $n=3$.

F3: Conditions (ii) and (iii) listed in Table 1 of $\mathbf{F 1}$ are equivalent, i.e.,

$$
\left[\mathbf{A}(t),\left(\int_{t_{o}}^{t} \mathbf{A}(\tau) d \tau\right)\right]=\left[\int_{t_{o}}^{t} \mathbf{A}(\tau) d \tau, \int_{t_{o}}^{t} \mathbf{A}(\tau) d \tau \mathbf{A}(t)\right] \Leftrightarrow\left[\mathbf{A}\left(t_{1}\right) \mathbf{A}\left(t_{2}\right)\right]=\mathbf{A}\left(t_{2}\right) \mathbf{A}\left(t_{1}\right) .
$$

The proofs of the above can be found in [6, Th 2.1], [8, Th 5.5], and [9, Th 2].

\section{F4: Matrix Exponentials and Commutativity of Constant and Time-Varying Matrices}

Given two square matrices $\mathbf{A}$ and $\mathbf{B}$, it follows that in general, [3], [12],

$$
\begin{aligned}
e^{\mathbf{A} t} \mathbf{B} & \neq \mathbf{B} e^{\mathbf{A} t}, \\
(14 a) & \\
e^{\mathbf{A} t} e^{\mathbf{B} t} & \neq e^{\mathbf{B} t} e^{\mathbf{A} t}, \\
e^{\mathbf{A} t} e^{\mathbf{B} t} & \neq e^{(\mathbf{A}+\mathbf{B}) \mathbf{t}} .
\end{aligned}
$$

Equality will hold if and only if $\mathbf{A}$ and $\mathbf{B}$ commute, i.e., $\mathbf{A B}=\mathbf{B} \mathbf{A}$. Further, if $\mathbf{A B}=\mathbf{B A}$, then $e^{\mathbf{A} t} e^{\mathbf{B} t}=e^{\mathbf{B} t} e^{\mathbf{A} t}=e^{\mathbf{A} t+B t} \Rightarrow e^{\mathbf{A} t}=e^{\mathbf{A} t+\mathbf{B} t-\mathbf{B} t}$. In addition, if $\mathbf{Y}$ is invertible, then $e^{\mathbf{Y} \mathbf{X Y}}=\mathbf{Y} e^{\mathbf{X}} \mathbf{Y}^{-1}[13]$.

\section{Analytical Solutions and Simulations of LTV Systems}

The analytical solutions of the Fundamental and State Transition Matrices and simulations of LTV systems together with Matlab Solutions will be investigated and compared in this section. It is known that the LTV system $\dot{\mathbf{x}}(t)=\mathbf{A}(t) \mathbf{x}+\mathbf{B}(t) \mathbf{u}$, where $\mathbf{A}(\mathrm{t})$ and $\mathbf{B}(\mathrm{t})$ are $(n-1)$ times consciously differentiable and $\operatorname{rank}[\mathbf{B}(\mathrm{t})]=\ell$, is controllable if the $n \times n \ell$ controllability matrix $C_{\mathrm{AB}}(t)$ defined by $[4$, Th $6.12 ; 6$, Th $2.5 ; 16]$

$$
C_{A B}(t)=\left[\begin{array}{l:l:l:l}
C_{o}(t) & C_{1}(t) & \cdots & C_{n-1}(t)
\end{array}\right]
$$

has $\operatorname{rank}\left[C_{A B}(t)\right]=n$, where

$$
C_{o}(t)=\mathbf{B}(t), \quad C_{i}(t)=-\mathbf{A}(t) C_{i-1}(t)+\dot{C}_{i-1}(t), \quad i=1,2, \cdots, n-1 .
$$

For LTI systems, (15) and (16) yield

$$
C_{A B}=\left[\begin{array}{l:l:l:l:l:l}
\mathbf{B} & -\mathbf{A B} & \mathbf{A}^{2} \mathbf{B} & -\mathbf{A}^{3} \mathbf{B} & \cdots & \mathbf{A}^{n-1} \mathbf{B}
\end{array}\right],
$$

which has the same rank as the standard controllability theorem of the pair $[\mathbf{A}, \mathbf{B}]$ given by

$$
\bar{C}_{A B}=\left[\begin{array}{l:l:l:l:l:l}
\mathbf{B} & \mathbf{A B} & \mathbf{A}^{2} \mathbf{B} & \mathbf{A}^{3} \mathbf{B} & \cdots & \mathbf{A}^{n-1} \mathbf{B}
\end{array}\right],
$$

i.e., the alternate minus sign in (17a) do not affect its rank and $\operatorname{rank}\left(C_{A B}\right)=\operatorname{rank}\left(\bar{C}_{A B}\right)$.

Example 1: Second-order LTV system [14], [15]

Consider the LTV system: 


$$
\dot{\mathbf{x}}=\mathbf{A}(t) \mathbf{x}=\left[\begin{array}{cc}
-6 t^{2} & 3 t^{5} \\
0 & -3 t^{2}
\end{array}\right] \mathbf{x}, \quad \mathbf{x}\left(t_{o}\right)=\mathbf{x}_{o},
$$

where $\mathbf{A}(t)$ is an upper triangular matrix. The primary objectives of this example are:

(1a): Solving for $\mathbf{x}(t)$, the fundamental matrix $\mathbf{X}(t)$, and the state transition matrix $\mathbf{\Phi}(t, 0)$;

(1b): Simulating the responses of $\mathbf{x}(t)$ in Matlab with normalized ICs: $x_{1}(0)=1$ and $x_{2}(0)=1$.

\section{Solution:}

(1a): First, we check the matrix commutativity properties of $\mathbf{A}(t)$ :

$$
\mathbf{A}\left(t_{1}\right) \mathbf{A}\left(t_{2}\right) \neq \mathbf{A}\left(t_{2}\right) \mathbf{A}\left(t_{1}\right) \quad \text { and } \quad \mathbf{A}(t) \mathbf{M}(t) \neq \mathbf{M}(t) \mathbf{A}(t),
$$

where $\mathbf{M}(t)=\int_{o}^{t} \mathbf{A}(\tau) d \tau$. Hence both conditions are not met, and $\mathbf{X}(t) \neq \exp \left(\int_{0}^{t} \mathbf{A}(\tau) d\right)$. The problem has been investigated in [14] and [15]. The solutions of Wu and Jain are listed below, respectively:

(i) $\mathrm{Wu}: \boldsymbol{\Phi}_{W u}(t, 0)=\left[\begin{array}{c:c}e^{-2 t^{3}} & \left.e^{-2 t^{3}}-e^{-t^{3}}+t^{3} e^{-t^{3}}\right) \\ 0 & e^{-t^{3}}\end{array}\right], \boldsymbol{\Phi}_{W u}(0,0)=\mathbf{I}_{2}$,

$\Rightarrow \quad \mathbf{x}(t)_{W u}=\left[\begin{array}{l}x_{1}(t)_{W u} \\ x_{2}(t)_{W u}\end{array}\right]=\left\{\begin{array}{l}e^{-2 t^{3}} x_{1}(0)+\left(e^{-2 t^{3}}-e^{-t^{3}}+t^{3} e^{-t^{3}}\right) x_{2}(0), \\ e^{-t^{3}} x_{2}(0),\end{array}\right.$

where (1-4) can also be obtained by the Method of Superposition Principle (MSP) via (10).

(ii) Jain: $\boldsymbol{\Phi}_{\text {Jain }}(t, 0)=\left[\begin{array}{c:c}e^{-2 t^{3}} & \frac{t^{3}}{2}\left(e^{-t^{3}}-e^{-2 t^{3}}\right) \\ 0 & e^{-t^{3}}\end{array}\right], \quad \boldsymbol{\Phi}_{\text {Jain }}(0,0)=\mathbf{I}_{2}$,

$\Rightarrow \quad \mathbf{x}(t)_{\text {Jain }}=\left[\begin{array}{l}x_{1}(t)_{\text {Jain }} \\ x_{2}(t)_{\text {Jain }}\end{array}\right]=\left\{\begin{array}{l}e^{-2 t^{3}} x_{1}(0)+\frac{t^{3}}{2}\left(e^{-t^{3}}-e^{-2 t^{3}}\right) x_{2}(0), \\ e^{-t^{3}} x_{2}(0),\end{array}\right.$

where the state transition matrices $\boldsymbol{\Phi}_{W u}(t, 0) \neq \boldsymbol{\Phi}_{\text {Jain }}(t, 0)$. It follows that

$$
\begin{aligned}
& \frac{\partial \boldsymbol{\Phi}_{W u}(t, 0)}{\partial t}=\mathbf{A}(t) \boldsymbol{\Phi}_{W u}(t, 0) \quad \Rightarrow \text { Wu's solution is correct, } \\
& \frac{\partial \boldsymbol{\Phi}_{\text {Jain }}(t, 0)}{\partial t} \neq \mathbf{A}(t) \boldsymbol{\Phi}_{\text {Jain }}(t, 0) \quad \Rightarrow \quad \text { Jain's solution is incorrect. }
\end{aligned}
$$

Specifically, from (1-4) and (1-6), it follows that

$$
x_{2}(t)_{W U}=x_{2}(t)_{J a i n}, \quad \text { (both components are correct), }
$$

while the error between $x_{1}(t)_{W u}$ and $x_{1}(t)_{J a i n}$ is given by

$$
e_{1}(t)=x_{1}(t)_{W u}-x_{1}(t)_{\text {Jain }}=\left[e^{-t^{3}}\left(\frac{t^{3}}{2}-1\right)+e^{-2 t^{3}}\left(\frac{t^{3}}{2}+1\right)\right] x_{2}(0) \quad \rightarrow 0 \quad \text { as } t \rightarrow \infty .
$$


Further, the error between $\boldsymbol{\Phi}_{W u}(t, 0)$ and $\boldsymbol{\Phi}_{\text {Jain }}(t, 0)$ is given by

$$
\mathbf{E}(t)=\boldsymbol{\Phi}_{W u}(t, 0)-\boldsymbol{\Phi}_{\text {Jain }}(t, 0)=\left[\begin{array}{c:c}
0 & e^{-2 t^{2}}-e^{-t^{3}}+\frac{1}{2} t^{3}\left(e^{-t^{3}}+e^{-2 t^{3}}\right) \\
\hdashline 0 & 0
\end{array}\right] \rightarrow 0 \quad \text { as } t \rightarrow \infty .
$$

The Matlab program using Matlab's EXPM command that yields (1-5) is listed below:

syms $\mathrm{t}$

$$
\begin{aligned}
& \mathbf{A}=\left[\begin{array}{lll}
-6 * \mathrm{t}^{\wedge} 2 & 3 * \mathrm{t}^{\wedge} 5 ; 0 & -3 * \mathrm{t}^{\wedge} 2
\end{array}\right]
\end{aligned}
$$

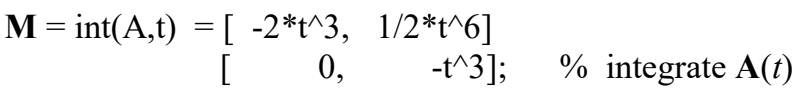

$$
\begin{aligned}
& \text { Xmatlab }=\operatorname{simplify}(\operatorname{expm}(\mathrm{M}))=\left[\exp \left(-2 * \mathrm{t}^{\wedge} 3\right), \quad-1 / 2 * \mathrm{t}^{\wedge} 3 * \exp \left(-\mathrm{t}^{\wedge} 3\right) *\left(-1+\exp \left(-\mathrm{t}^{\wedge} 3\right)\right)\right] \\
& \text { [ } \left.0, \quad \exp \left(-t^{\wedge} 3\right)\right] \text {, }
\end{aligned}
$$

which is identical to (1-5)).

(1b): The responses of the analytical solutions $\mathbf{x}(t)_{W u}$ and $\mathbf{x}(t)_{J a i n}$ given by (1-4) and (1-6), respectively, are plotted in Figure 1-1. For comparisons, simulations of the time-varying ODE (1-1) using Matlab's ODE45 are also plotted. All the plots in Figure 1-1 agree with the observations given by (1-10) and (1-11). Hence, based on the matrix differential equations $\frac{\partial \boldsymbol{\Phi}_{W u}(t, 0)}{\partial t}=\mathbf{A}(t) \boldsymbol{\Phi}_{W u}(t, 0)$ and $\frac{\partial \boldsymbol{\Phi}_{\text {Jain }}(t, 0)}{\partial t} \neq \mathbf{A}(t) \boldsymbol{\Phi}_{\text {Jain }}(t, 0)$ given by (1-7) and (1-8), the error equation given by (1-10), and all the simulation results, we conclude that $\mathrm{Wu}$ 's method yields the correct solution to $\dot{\mathbf{x}}=\mathbf{A}(t) \mathbf{x}$ and Jain's solution solutions are incorrect. Recall that Matlab will yield correct solutions if $\mathbf{A}(t)$ given in (1-1) is a commutative matrix.




Figure 1-1: Responses of $\mathbf{x}_{W u}(t)$ given by (1-4) and $\mathbf{x}_{\text {Jain }}(t)$ given by (1-6) with $x_{1}(0)=1$ and $x_{2}(0)=1$, and the error $e_{1}(t)$ given by (1-10). All the solutions decay to zero as $t \rightarrow \infty$.

\section{Example 2: Design of Feedback Control for LTV System}

Consider the LTV system described by [16]

$$
\dot{\mathbf{x}}=\mathbf{A}(t) \mathbf{x}+\mathbf{B}(t) u=\left[\begin{array}{cc}
0 & -1-\exp (-t) \\
1 & -\exp (-t)
\end{array}\right] \mathbf{x}+\left[\begin{array}{l}
0 \\
1
\end{array}\right] u,
$$

where the author's design objective was to change (2-1) to a canonical form, while our objectives focus on (2a) and (2b) below:

(2a): Modifying the structure of the non-commutative open-loop system matrix $\mathbf{A}(t)$ in (2-1) by feedback control such that the resulting closed-loop system matrix $\mathbf{A}_{c l}(t)=\mathbf{A}(t)-\mathbf{B}(t) \mathbf{K}(t)$ is commutative and triangular.

(2b): Determining the finite-sum solutions for $\mathbf{X}_{c l}(t)$ and $\mathbf{\Phi}_{c l}\left(t, t_{o}\right)$ obtained in (2a).

\section{Solution:}

(2a): Step 1: Check the controllability matrix of the pair $[\mathbf{A}(t), \mathbf{B}(t)]$ given by (15) and (16):

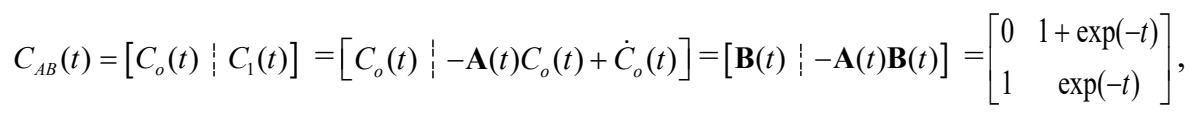

which yields the determinant $\operatorname{det}\left(C_{A B}(t)\right)$ of $\left(C_{A B}(t)\right)$ as

$$
\begin{aligned}
& \operatorname{det}\left(C_{A B}(t)\right)=-1-\exp (-\mathrm{t}) \neq 0 \text { for all } t \\
& \Rightarrow \quad \operatorname{rank}\left[C_{A B}(t)\right]=2 \text { for all } t \Rightarrow \text { LTV system }(2.1) \text { is controllable for all } t .
\end{aligned}
$$

Step 2: Choose $A_{c l}$ as

$$
\mathbf{A}_{c l}(t)=\left[\begin{array}{cc}
0 & -1-\exp (-t) \\
0 & 0
\end{array}\right],
$$

which is commutative and triangular.

Step 3: Determine the resulting control gain matrix $\mathbf{K}(t)$ : We have

$$
\begin{array}{rlrl} 
& \mathbf{A}_{\mathrm{cl}}(t) & =\mathbf{A}(t)-\mathbf{B}(t) \mathbf{K}(t) \Rightarrow \mathbf{B}^{T}(t) \mathbf{B}(t) \mathbf{K}(t)=\mathbf{B}^{T}(t)\left[\mathbf{A}(t)-\mathbf{A}_{c l}(t)\right] \\
\Rightarrow & \mathbf{K}(t)=\left[\mathbf{B}^{T}(t) \mathbf{B}(t)\right]^{-1}\left[\mathbf{A}(t)-\mathbf{A}_{c l}(t)\right]=\left[\begin{array}{ll}
1 & -\exp (-t)]
\end{array}\right.
\end{array}
$$

which yields $k_{1}(t)=1$ and $k_{2}(\mathrm{t})=-\exp (-t)$. Further, (2-5) yields

$$
(\mathbf{M}(t))^{2}=\mathbf{0},
$$

where

$$
\mathbf{M}(t)=\int_{o}^{t} \mathbf{A}_{c l}(\tau) d \tau=\left[\begin{array}{cc}
0 & -t+\mathrm{e}^{-t} \\
0 & 0
\end{array}\right] .
$$

(2b): Since $\mathbf{A}_{c l}(t)$ is commutative, the fundamental matrix $\mathbf{X}_{c l}(t)$ has the general form 


$$
\mathbf{X}_{c l}(t)=\mathrm{e}^{\int^{t}{ }^{A_{d}(t) d \tau}}=\sum_{k=0}^{\infty} \frac{1}{k !}\left(\int_{0}^{t} \mathbf{A}_{c l}(\tau) d \tau\right)^{k}=I_{2}+\mathbf{M}(t)+\frac{1}{2 !} \mathbf{M}^{2}(t)+\frac{1}{3 !} \mathbf{M}^{3}(t)+\cdots
$$

which, in view of (2-7), becomes a finite-sum solution given by

$$
\mathbf{X}_{c l}(t)=\mathbf{I}_{2}+\mathbf{M}(t)=\left[\begin{array}{cc}
1 & -t+\mathrm{e}^{-t} \\
0 & 1
\end{array}\right], \quad \mathbf{X}_{c l}(0)=\left[\begin{array}{ll}
1 & 1 \\
0 & 1
\end{array}\right] .
$$

Using (2-10), the transition matrix $\boldsymbol{\Phi}_{c l}(t, 0)$ is obtained as

$$
\boldsymbol{\Phi}_{c l}(t, 0)=\mathbf{X}_{c l}(t) \mathbf{X}_{c l}^{-1}(0)=\left[\begin{array}{cc}
1 & -1-t+e^{-t} \\
0 & 1
\end{array}\right], \quad \boldsymbol{\Phi}_{c l}(t, 0)=\mathbf{I}_{2} .
$$

Equations (2-10) and (2-11) satisfy, respectively, the matrix differential equations:

$$
\begin{aligned}
& \dot{\mathbf{X}}_{c l}(t)=\mathbf{A}_{c l}(t) \mathbf{X}_{c l}(t), \quad \mathbf{X}_{c l}(0), \\
& \dot{\boldsymbol{\Phi}}_{c l}(t, 0)=\mathbf{A}_{c l}(t) \mathbf{\Phi}_{c l}(t, 0), \quad \mathbf{\Phi}_{c l}(0,0)=\mathbf{I}_{2},
\end{aligned}
$$

which confirm that $\mathbf{X}_{c l}(t)$ and $\boldsymbol{\Phi}_{c l}(t, 0)$ solve (2-12) and (2-13), respectively.

Example 3: Design of feedback control system with prescribed proprieties

Consider the 3rd-order LTV system described by

$$
\dot{\mathbf{x}}=\mathbf{A}(t) \mathbf{x}+\mathbf{B}(t) u=\left[\begin{array}{ccc}
0 & 1 & t^{2} \\
0 & -2 t & -t \\
0 & -2 t & 1-t
\end{array}\right] \mathbf{x}+\left[\begin{array}{l}
0 \\
1 \\
1
\end{array}\right] u .
$$

(3a): Design a feedback control system for (3-1) such that the resulting closed-loop system matrix $\mathbf{A}_{c l}(t)=\mathbf{A}(t)-\mathbf{B}(t) \mathbf{K}(t)$ is commutative and triangular.

(3b): Determine the analytical solutions for the fundamental matrix $\mathbf{X}_{c l}(t)$ and state transition matrix $\mathbf{\Phi}_{c l}\left(t, t_{o}\right)$.

\section{Solution:}

(3a): Step 1: Check the controllability matrix $C_{A B}(t)$ of the pair $[\mathbf{A}(t), \mathbf{B}(t)]$ given by (15) and (16):

$$
C_{A B}(t)=\left[\begin{array}{l:l:l}
C_{o}(t) & C_{1}(t) & C_{2}(t)
\end{array}\right]=\left[\begin{array}{l:l:l}
C_{o}(t) & -\mathbf{A}(t) C_{o}(t)+\dot{C}_{o}(t) & -\mathbf{A}(t) C_{1}(t)+\dot{C}_{1}(t)
\end{array}\right],
$$

where

$$
\begin{aligned}
& C_{\mathrm{o}}(t)=\mathbf{B}, \\
& C_{1}(t)=-\mathbf{A}(t) C_{o}(t)+\dot{C}_{o}(t)=\left[\begin{array}{c}
-1-t^{2} \\
3 t \\
3 t-1
\end{array}\right] \\
& C_{2}(t)=-\mathbf{A}(t) C_{1}(t)+\dot{C}_{1}(t)=\left[\begin{array}{c}
-5 t-t^{2}(3 t-1) \\
2+5 t^{2}+t(3 t-1) \\
2+5 t^{2}+(t-1)(3 t-1)
\end{array}\right] .
\end{aligned}
$$


Substituting (3-3), (3-4) and (3-5) into (3-2) and computing the determinant $\operatorname{det}\left(C_{A B}\right)$ of $C_{A B}$ yields $\operatorname{det}(\mathrm{CAB})=1+2 \mathrm{t} \neq 0$,

$\Rightarrow \quad \operatorname{rank}\left[C_{A B}(t)\right]=3$ for all $t \Rightarrow$ LTV system (3.1) is controllable for all $t$.

Step 2: Choose $\mathbf{A}_{c l}(t)$ as

$$
\mathbf{A}_{c l}(t)=\left[\begin{array}{ccc}
0 & 1 & t^{2} \\
0 & 0 & -1 \\
0 & 0 & 0
\end{array}\right] .
$$

Step 3: Determine the resulting control gain matrix $\mathbf{K}(t)$ : We have

$$
\begin{aligned}
\mathbf{A}_{c l}(t) & =\mathbf{A}(t)-\mathbf{B}(t) \mathbf{K}(t) \quad \Rightarrow \quad \mathbf{B}^{T}(t) \mathbf{B}(t) \mathbf{K}(\mathrm{t})=\mathbf{B}^{T}(t)\left[\mathbf{A}(t)-\mathbf{A}_{c l}(t)\right], \\
\Rightarrow \quad & \mathbf{K}(t)=\left[\mathbf{B}^{T}(t) \mathbf{B}(t)\right]^{-1} \mathbf{B}^{T}(t)\left[\mathbf{A}(t)-\mathbf{A}_{c l}(t)\right],
\end{aligned}
$$

which yields $k_{1}=-1, \quad k_{2}=-2 t$ and $k_{3}=1-t$. Further, (3-7) yields

$$
\mathbf{M}^{3}(t)=\left[\begin{array}{lll}
0 & 0 & 0 \\
0 & 0 & 0 \\
0 & 0 & 0
\end{array}\right],
$$

where

$$
\mathbf{M}(t)=\int_{o}^{t} \mathbf{A}_{c l}(\tau) d \tau=\left[\begin{array}{ccc}
0 & t & \frac{1}{3} t^{3} \\
0 & 0 & -t \\
0 & 0 & 0
\end{array}\right] .
$$

(3b): Since $\mathbf{A}_{c l}(t)$ is commutative, the fundamental matrix $\mathbf{X}_{c l}(t)$ has the general form

$$
\mathbf{X}_{c l}(t)=\mathrm{e}^{\int_{o}^{t} \mathbf{A}_{c l}(\tau) d \tau}=\sum_{k=0}^{\infty} \frac{1}{k !}\left(\int_{o}^{t} \mathbf{A}_{c l}(\tau) d \tau\right)^{k}=I_{2}+\mathbf{M}(t)+\frac{1}{2 !} \mathbf{M}^{2}(t)+\frac{1}{3 !} \mathbf{M}^{3}(t)+\cdots
$$

which, in view of (3-10), becomes a finite-sum solution given by

$$
\mathbf{X}_{c l}(t)=\mathbf{I}_{3}+\mathbf{M}(t)+\frac{1}{2 !} \mathbf{M}^{2}(t)=\left[\begin{array}{ccc}
1 & t & \frac{1}{3} t^{3}-\frac{1}{2} t^{2} \\
0 & 1 & -t \\
0 & 0 & 1
\end{array}\right], \quad \mathbf{X}_{c l}(t, 0)=\mathbf{I}_{3} .
$$

Using (3-12), the transition matrix $\boldsymbol{\Phi}_{c l}(t, 0)$ is obtained as, for $\mathbf{X}_{c l}(t, 0)=\mathbf{I}_{3}$,

$$
\mathbf{\Phi}_{c l}(t, 0)=\mathbf{X}_{c l}(t), \quad \boldsymbol{\Phi}_{c l}(t, 0)=\mathbf{I}_{3} .
$$

Equations (3-13) and (3-14) satisfy, respectively, the matrix differential equations:

$$
\begin{aligned}
& \dot{\mathbf{X}}_{c l}(t)=\mathbf{A}_{c l}(t) \mathbf{X}_{c l}(t), \quad \mathbf{X}_{c l}(0), \\
& \dot{\boldsymbol{\Phi}}_{c l}(t, 0)=\mathbf{A}_{c l}(t) \boldsymbol{\Phi}_{c l}(t, 0), \quad \boldsymbol{\Phi}_{c l}(0,0)=\mathbf{I}_{2},
\end{aligned}
$$

which confirm that $\mathbf{X}_{c l}(t)$ and $\boldsymbol{\Phi}_{c l}(t, 0)$ solve (3-13) and (3-14), respectively.

\section{Conclusions}


The determination of the fundamental and state transition matrices and the design of feedback control of linear time-varying (LTV) systems of the form $\dot{\mathbf{x}}=\mathbf{A}(t) \mathbf{x}+\mathbf{B}(t) \mathbf{u}$ were investigated. One of the main goals was to render the closed-loop system matrix $\mathbf{A}_{c l}(t)$ commutative and triangular, thereby facilitating the determinations of the closed-form analytical solutions for $\mathbf{X}_{c l}(t)$ and $\boldsymbol{\Phi}_{c l}\left(t, t_{o}\right)$ associated with $\mathbf{A}_{c l}(t)$. It should be emphasized that this is different from a pole-placement design problem where the goal is to place the poles of $\mathbf{A}_{c l}(t)$ to achieve desirable closed-loop properties, whereas the goals in this paper were to make $\mathrm{A}_{c l}(\mathrm{t})$ diagonal and triangular. Examples were given to demonstrate the analysis and design. Matlab solutions were provided as well. Future research will address the designs of observers and unknown-input observers (UIO).

\section{References}

[1] Zhu, J., Morales, C.H., "On Linear Ordinary Differential Equations with Functionally Commutative Coefficient Matrices", Linear Algebra and Its Applications, 170:81-105, 1992.

[2] Cleve Moler, Charles Van Loan, "Nineteen Dubious Ways to Compute the Exponential of a Matrix, Twenty-Five Years Later", SIAM REVIEW, Society for Industrial and Applied Mathematics, Vol. 45, No. 1, 46 pages.

[3] Kailath, T., Linear Systems, New Jersey: Prentice Hall, 1980.

[4] Chen, C.T., Linear Systems and Design, 4th ed., New York: Oxford University Press, 2013.

[5] Taha, A.F., "Computation of State Transition Matrix, Module 04 - Linear Time-Varying Systems".

[6] "Chapter 2, Analysis and control of linear time-varying systems", https://www.control.tf.unikiel.de/en/teaching/summer-term/nonlinear-controlsystems/fileadmin/ncs_ss20_chap2.

[7] Martin, J.F.P., "Somre results on matrices which commute with their derivatives', SIAM J. AFFL. MATH, Vol. 18, No. 8, 1967.

[8] Wiberg, D.M., Theory and Problems of State Space and Linear Systems, New York, McGrawHill, Inc., 1971.

[9] Kamizawa, T., "On Functionally Commutative Quantum Systems", Faculty of Physics, Astronomy and Informatics, Nicolaus Copernicus University, Toru'n, Poland, 2018.

[10] Dahleh, M., Dahleh, M., Verghese, G., "Chapter 11, Lectures on Dynamic Systems and Control", MIT6_241JS11.

[11] Bamieh, B., "Lecture 5: Continuous-Time Linear State-Space Models", University of California, Fall 1999.

[12] Gustafson, 2250-Systems of Differential Equations, Chapter 11, 69 pages.

[13] Matrix Calculus, Wikipedia.

[14] Wu, M.Y., "A new method of computing the state transition matrix of linear time-varying systems", Proceedings of the IEEE International Symposium on Circuits and Systems, San Francisco, 269-272, 1974.

[15] Jain, Vanita, and Lande, B.K., "Computation of the transition matrix for general linear-varying systems", International Journal of Engineering \& Technology (IJERT), Vol. 1, Issue 6, 2012.

[16] Kamen, Edward Walter, "Chapter 3, Fundamentals of Linear Time-Varying Systems", Georgia Institute of Technology, December 10. 